DOI: $10.2478 /$ auseb-2014-0007

\title{
Socio-Economic Status and the Structural Change of Dietary Intake in Hungary
}

\author{
Zoltán BAKUCS
}

Centre for Economic and Regional Studies, Hungarian Academy of Sciences, Corvinus University of Budapest

E-mail: zoltan.bakucs@krtk.mta.hu

\section{Imre FERTŐ}

Centre for Economic and Regional Studies, Hungarian Academy of Sciences, Corvinus University of Budapest

E-mail: imre.ferto@krtk.mta.hu

\section{Cristina Galamba MARREIROS \\ Centro de Estudos e Formação Avançada em Gestão e Economia (CEFAGE-UE), University of Évora, Portugal \\ E-mail: cristina@uevora.pt}

\begin{abstract}
Typically, big changes in the economic system lead to alterations on families' disposable income and thus on their spending for different types of products, including food. These may imply in the long run a structural modification of the population's diet quality. After the fall of the socialist system, in the past two decades, Central and Eastern European countries, including Hungary, went through a profound and sometimes difficult transition of their political and economic systems, shifting from a centralized plan to an open-market economy, and, perhaps more importantly, the European Union integration. Economic change in lower-income and transitional economies of the world appears to coincide with increasing rapid social change. With respect to nutrition, there is evidence that these countries are changing their diets and that changes seem to happen at a faster pace than ever before (e.g. Ivanova et al., 2006). In this paper, we analyse the evolution of Hungarian dietary patterns based on socio-economic status (SES) data between 1993 and 2007. Data allows defining and profiling several clusters based on aggregated consumption data, and then inspecting the influence of SES variables using OLS and multinomial logit estimations.
\end{abstract}

Keywords and phrases: Transition economy, food consumption patterns, cluster analysis, logit analysis.

JEL Classification: I15, C25, D1 


\section{Introduction}

In most Central and Eastern European post-communist economies, food expenditures constitute the second largest expenditure position for private households (overshadowed only by expenditures for housing). A significant welfare loss due to increased nutrition-related expenditures was recorded in the transition period (see Huffman 2005 for the Polish example). Nevertheless, food expenditure shares as well as absolute expenses per household are declining. In 1995, for Hungarian households, this represented $23.3 \%$ declining to $17.50 \%$ on average by 2008 versus $14.5 \%$ and $12.7 \%$ for EU-27, respectively. A comparison of consumption behaviour between East and West Germany reveals a clear tendency of convergence for most products (Grings, 2001).

Moreover, in a study of food expenditures across 47 countries, Regmi et al. (2008) found significant convergence of consumption patterns for total food, cereals, meats, seafood, dairy, sugar and confectionery, caffeinated beverages and soft drinks. According to the authors, this convergence reflects consumption growth in middle-income countries - to which Hungary also belongs - due to the rapid modernization of their food delivery systems as well as to global income growth. Quoting Regmi and Gehlhar (2005), this study concludes that consumers in developing countries have used their growing incomes to upgrade diets, increase their demand for meats, dairy products and other higher-value food products.

Several studies, however, (e.g. Irala-Estévez et al., 2000; James et al., 1997; Arija et al., 1996; Ross et al., 1996) show that there are large variations between individuals with respect to the quantity and quality of the food consumed. Despite the fact that lower-income consumers make bigger changes in food expenditures as their income levels change (Seale et al., 2003), there is empirical evidence (e.g. Hulshof et al., 2003; Cavelaars et al., 1997; Adler et al., 1994; Hoeymans et al., 1996) that in most European countries there are still great disparities in nutrition and health with respect to socio-economic status (SES).

In general, less educated and lower-income groups appear to consume less healthy diets (Hulshof et al., 2003). According to the studies of Dowler et al. (1997) and James et al. (1997), poverty and low income may restrict the ability to buy food on the basis of health and limit access to healthy food. According to Hulshof et al. (2003), particularly in the North and West of Europe, a higher SES is associated with a greater consumption of low-fat milk, fruit and vegetables (e.g. Irala-Estevez et al., 2000). Additionally, those with higher education tend to consume less fat and oil but more cheese (Hulshof et al., 2003; Roos et al., 1999). Prattala et al. (2003) confirmed this finding, concluding that higher and lower socio-economic groups have different sources of saturated fats.

A previous research also concluded that consumers with a higher educational level tend to be more aware of the characteristics of a healthy diet (Margetts 
et al., 1997) and have more knowledge about food items which are healthier (Martinez-Gonzales et al., 1998; Hjartaker and Lund, 1998; Margetts et al., 1997). Although not directly related to this study, the issue of perceived food healthiness or the subjective diet awareness needs to be mentioned (see, for example, Provencher et al., 2008 or Carels et al., 2007). Although Hulshof et al. (2003) state that this might partly explain the differences in food consumption between SES classes, the differences in food consumption patterns between SES may also be explained by the findings of Prattala et al. (2003) that higher social classes prefer modern foods whilst lower classes traditional foods. This conclusion is in line with Grignon's (1999) emphasizing that higher social classes consume more food items, indicating an increasing trend compared to lower classes. According to the authors, these findings are explained by the Bourdieu's theory that the socio-economically better-off are the first to adopt new food habits (Bourdieu, 1989).

To further understand the role of SES in food consumption, in this paper, we analyse the differences in dietary intake between adults with different socioeconomic statuses (SES) and trends over time. Using family food consumption household data from the beginning of the transition period (1993) and from after the EU accession (2007), we analyse the declared consumption of the main food groups, looking into the differences of the diets of consumer groups with different SES in Hungary. This study allows for the analysis of the convergence of the Hungarian diet with the diets of other European countries and the identification of possible measures to improve the dietary intake of consumers.

This paper is organized as follows: after this introduction, a brief description of the research methodology is presented; the empirical results of the study are discussed in Section 3, followed by summary, conclusions and recommendations. The conclusions stress the main findings and discuss implications for food policies in what concerns the improvement of the Hungarian diet. Several directions for possible further research in this topic are outlined at the end of this study.

\section{Data and Methodology}

The Hungarian Household Budget Survey (HBS) has been conducted annually by the Hungarian Central Statistical Office since 1993. The survey covers the Hungarian population living in private households. The unit of sampling is the dwelling and the unit of observation is the household. The survey contains 7,000 to 10,000 households annually. The survey is partly based on monthly household records and partly on post facto annual interviews, providing detailed information both on income and the structure of expenditures. Own consumption of self-produced food and beverages and net farm revenue are also reported. 
The empirical analysis employs three multivariate techniques. First, cluster analysis is used to group households according to their food consumption habits. Then, a more detailed multivariate regression analysis follows, where healthy and less healthy food consumption habits are regressed on variables defining SES. Thus, dependent variables include quantities of fat, sugar, alcohol, various meats, fruit and vegetables consumption, whilst independent variables include household size, age and education of the household head, location, income, employment and quality of house/flat (number of rooms, existence of bathrooms, etc.).

Several different measures of socio-economic status, such as education, location, house characteristics, were examined in this study. The aim was to compare the direction and magnitude of associations for each measure of socioeconomic status with the fruit and vegetable intake. Educational level, cultural expenditures or the geographical location (Budapest or other large cities) may have important influences on the socio-economic status. Higher levels of education may increase the ability to obtain or to understand health-related information in general, or dietary information in particular, needed to develop health-promoting behaviours and beliefs with respect to food consumption habits. Analyses, which have taken into account education, occupation, income and employment status alike, have shown that education is usually the strongest determinant of the socio-economic differences. The other socio-economic variables have a similar but weaker effect than education (Roos et al., 1996).

Multivariate regressions differ from multiple regressions in that several dependent variables are jointly regressed on the same explanatory variables. Although direct comparison of 1993 and 2007 regression coefficients should be done with care since variables are not entirely the same in the two databases, the analysis gives insight not only into consumption and dietary habit differences across SES groups, but also into their change in time. The latter is a rather important issue in the postcommunist economies, where the economic transformations that started in 1990 had a deep impact upon population purchase power, income and, indeed, food consumption habits (Ivanova et al., 2006). Finally, a multinomial logit analysis is performed. Using information from the first part of the empirical analysis, cluster numbers employed as dependent variables are regressed upon SES variables.

\section{Empirical Results}

\section{Descriptive Statistics}

First, a number of SES variables were selected for the analysis. The descriptive statistics of the most important ones are presented in Table 1: education of household head $(E d u)$, income of household (InC: monthly total personal income 
of household head in 1993 and the deciles the household belongs to based on net income per person for 2007), location (Loc: 1 - Budapest, 2 - major city, 3 - town, 4 - village), number of people in the household (Num), number of larger than $12 \mathrm{~m}^{2}$ rooms and number of 4 to $12 \mathrm{~m}^{2}$ rooms in the household (R1 and $R 2$, respectively), bathroom and toilet facilities in the household $(B R)$, agricultural income (AInC) and cultural expenditures (Cult). Nine aggregated food consumption variables were created, based on individual food item consumption data. Number of observations, mean values, standard deviation, minimum and maximum values of the aggregated variables for 1993 and 2007 are presented in tables 2 and 3 , respectively.

The last column of tables 2 and 3 shows the percentage of aggregated consumption variables within total food consumption (sum of all 9 categories). Surprisingly, the structure of food consumption remained almost unchanged during the 14 years time span. There is more consumption of red and white meats in 2007, but a shift from animal to vegetable fats may also be observed. The share of vegetables in total consumption had been massively reduced by 2007; however, the share of fruit consumption remained stable. With the increase of the 2007 carbohydrates and alcohol intakes, one may conclude that dietary habits in Hungary shifted towards less healthy consumption patterns.

\section{Cluster Analysis of Food Consumption Patterns}

Cluster analysis was applied as a two-stage process to the following 9 aggregated food intake variables: red meats, white meats, egg and milk products, animal fats, vegetable fats, vegetables, fruits, carbohydrates and alcohol. In the first stage, a hierarchical analysis was employed to provide an indication of the appropriate number of clusters. Hair et al. (1998) suggests a procedure based upon the inspection of the distance information from the agglomeration schedule. Following this procedure, the appropriate number of clusters is suggested at the stage where there is a 'large' increase in the distance measure, indicating that a further merger would result in decrease in homogeneity. However, Hair et al. (1998) also point out that 'the selection of the final cluster solution requires substantial researcher judgement and is considered by many to be too subjective'. Following the hierarchical analysis, and the exclusion of outliers in both databases, the K-Means optimization procedure was employed - together with the consideration of relative cluster size and the desire for parsimony - to generate a three-cluster solution for 1993 and a two-cluster solution for 2007. Information about cluster membership, in the form of a nominal cluster identity variable, and distance to the cluster centre were saved for posterior analysis.

F-tests were performed to the cluster variables. These are based upon differences between clusters, on the basis of a null hypothesis that average variable scores 
for each cluster are equal against an alternative hypothesis that they are not. Results indicate that the 9 variables have significantly different patterns between groups. Therefore, the criteria used to cluster consumers can be considered meaningful. The next step of the analysis is to profile the clusters. A profile of each of the groups is established from the mean of the food intake variables and from the identification of the SES variables for which there are significant differences between groups at a $5 \%$ level of significance on the basis of a chisquare contingency test for nominal variables, and an F-test for metric variables.

Of the 3 clusters found in the 1993 panel, Cluster 3 is the biggest cluster with more than half of the households $(62.3 \%)$, followed by Cluster $2(34.5 \%)$ and by a quite small Cluster 1 with only $2.7 \%$ of observations. Analysing the profiles of the clusters, significant differences at the $5 \%$ level were found in all food intake variables and in SES variables in analysis, except for the amount spent for culture (proxied by concert and theatre expenditures).

Cluster 3 has the lowest scores in all food intake variables as well as in the income variable, partly explained by the fact that families of this cluster are smaller (average size of 2.32 members vs. 3.28 in Cluster 2 and 3.65 in Cluster 1). These families live in smaller houses (flats) than families in the other two clusters and spend relatively less on books. Additionally, they tend to live relatively more in Budapest and other cities (27.5\% vs. $16.3 \%$ in Cluster 2 and $10.8 \%$ in Cluster 1) and to have a woman as head of the household (34.7\% vs. $10.8 \%$ in Cluster 2 and $8.2 \%$ in Cluster 1). The head of the household is relatively older than in the other two clusters (54.6 vs. 50.22 in Cluster 2 and 47.93 in Cluster 1). With respect to education level, the profile of this cluster is somewhat mixed, since it has the highest proportion of people with less than 8 years of school (25.25 vs. $15.8 \%$ in Cluster 2 and $14.8 \%$ in Cluster 1) and, at the same time, with a university or college education (8.6\% vs. $7.1 \%$ in Cluster 2 and $5.1 \%$ in Cluster 1$)$.

As it can be understood form the previous paragraph, Cluster 1 is more different from Cluster 3 than Cluster 2 in terms of both food intake and socio-economic profile. This rule does not apply to the consumption of fruits, where Cluster 2 has the highest score, followed by Cluster 1 and then Cluster 3. The same is true for the number of rooms in the household smaller than $12 \mathrm{~m}^{2}$, where the mean value of Cluster 2 is higher. It is also important to notice that Cluster 1 is the cluster with a higher proportion of people living in the countryside $(59.7 \%$ vs. $52.1 \%$ in Cluster 2 and $40.5 \%$ in Cluster 3) with 8 to 10 years of school $(36.7 \%$ vs. $31.5 \%$ in Cluster 2 and $29.6 \%$ in Cluster 3) and with a man as the head of the household (91.8\% vs. $89.2 \%$ in Cluster 2 and $65.3 \%$ in Cluster 3 ).

The profiles of the 2007 clusters show that Cluster 1 is the smallest one, with $26.5 \%$ of observations. The mean value of the food intake variables is always higher in this cluster. When compared to Cluster 2, consumers in Cluster 1 are characterized by relatively lower education levels and live relatively more in 
rural areas and small cities (71.8\% vs. 53.9\% in Cluster 2). They live in bigger households and spend more on education, culture and holidays. These may be explained by the fact that they tend to have bigger families than consumers in Cluster 2 (mean value of 3.41 vs. 2.3). The per capita total income is relatively lower in this cluster - the percentage of observations in deciles 1 to 7 is significantly higher for this group. The head of the household tends to be younger than in Cluster 2 (mean value of 50.3 vs. 52 ) and it is a man (83.3\% versus $62.3 \%$ ).

To conclude, it can be stated that this cluster is composed of more traditional families, with relatively lower per capita income, that live in the countryside, have more children and a relatively young man as head of the household, with a medium level of education.

\section{Regression Analysis of Food Consumption on SES Variables}

OLS regressions of aggregated consumption variables on cluster data and SES variables are performed next. Table 4 presents regression coefficients and corresponding significance levels for 1993, whilst Table 5 does so for 2007.

For 1993, the coefficients of determination (adjusted $\mathrm{R}^{2}$ ) vary considerably between regressions, from $6 \%$ (fruits, alcohols, vegetable fats and animal fats) to $30 \%$ (carbohydrates) or even 66\% (egg and milk products). Similarly dispersed, albeit somewhat higher $\mathrm{R}^{2}$ values were obtained for 2007, ranging from $7 \%$ (alcohols) to $15-20 \%$ for meat, vegetable and fruit products, 48\% (carbohydrates) and $64 \%$ (egg and milk products).

Explanatory variables are generally highly significant and their sign is persistent from 1993 to 2007 regressions. For 1993, the cluster analysis revealed that households in Cluster 1 consume the most, followed by clusters 2 and 3. For the 2007 data, Cluster 1 consumes more than Cluster 2. The finding is reflected by the negative coefficients of the cluster variable in every regression for both 1993 and 2007. The gender variable is negative for all categories, implying that households managed by women consume less. Education, coded from 1 (less than 8 classes) to 8 (PhD), significantly reduces consumption except for vegetable fats and fruits, possibly suggesting more health-conscious eating habits for highly educated households.

For 1993, the income variable is only significant (positive) for red meats, vegetable fats, fruits and alcohol, the more expensive food categories. For 2007, the income variable is significantly positive for all food categories except the cheaper and possibly less income-sensitive ones, such as animal fats and carbohydrates. The higher number of food categories, where the variable is significant in 2007 compared to 1993, might suggest the growing importance of household income when purchasing food, i.e. the increase of the food demands' income elasticity coefficient. Location (from 1: Budapest to 4: village) has significantly positive 
(e.g. white meats, egg-milk products, animal fats and carbohydrates for 1993) and negative effects (vegetable fats, consumed more in larger localities) depending on food category. For 2007, the variable suggests increased consumption of most food categories in smaller localities compared to bigger ones, with the exception of vegetables, fruits and alcohols. With the exception of alcohols (negative for 1993, not significant for 2007), the number of household members positively influence all aggregated food categories. The negative sign of alcohols indicates that households with larger families (more children) tend to spend less on such items. The number of smaller and larger rooms (R1 and R2) is generally significant and increases the consumption of all food variables. The picture is less obvious for the number of bathrooms/toilets in the household. Agricultural income seems to be an important determinant in both years, with mostly significant positive coefficients (correlation coefficient between net income and agricultural sales/ income is close to 0). There is an extra variable included in the 2007 regressions, not available for the 1993 data: the cultural, artistic expenditures (cult). With the exception of alcohols and fruits, where it is significantly positive, it has negative effects upon all other food consumption categories. Perhaps those willing to spend more on culture, arts and, ultimately, going out, tend to consume more alcohol in and outdoors, and, at the same time, reduce their intake of other food items.

\section{Analysis of Cluster Selection on SES Variables}

A multinomial logit analysis is run for 1993 with the dependent variable being the cluster (1,2 or 3). For the 2-cluster solution in 2007, a logit regression is performed. Results for 1993 and 2007 are presented in tables 6 and 7, respectively.

The coefficients of the multinomial logit regression fit the cluster profiles presented in Section 3.2: Cluster 3, the base is the cluster with the lowest food intake, smaller houses ( $R 1, R 2$ positive in clusters 1 and 2 versus the base), live mostly in Budapest or bigger cities (positive coefficient for location in both clusters vs. Cluster 3), smaller families (variable Num positive). In a similar fashion, those in Cluster 1 are more likely to live in rural areas than those in Cluster 2 or 3 (positive location and agricultural income coefficients), and they are more likely to have a man as household head.

\section{Conclusions}

Our results emphasize the major post-1990 socio-economic changes in the Hungarian society. Dietary intakes vary considerably across SES and also in time. Similarly to the Bulgarian findings of Ivanova et al. (2006), a general deterioration of post-transition dietary habits is observed; however, some SES groups managed to shift their food consumption towards healthier intake patterns. 
Due to increasing household incomes and the openness of the Hungarian economy, the consumption patterns of Hungarian families tend to converge. While in 1993 three distinct clusters of food consumption patterns were identified, in 2007, only two groups could be found. Nevertheless, the great majority of the variables that used to define the SES have a significant impact on food consumption patterns in both years, confirming previous studies on this issue and showing that, besides income, other variables, such as education, gender, type of household etc., are also pertinent to understanding food consumption behaviour.

It is also important to emphasize and understand the change of food consumption patterns in Hungary after the economic transition and the EU accession. As expected, with a growing economy, people spend more on meat products (both red and white), converging towards the EU average. Concerning health-conscious food consumption behaviours however, mixed results were obtained. On the one hand, there is a tendency to replace animal fats with vegetable fats, along with increased fruit consumption. On the other hand, there is a sharp decrease of the share of vegetables in total consumption and an increase of the share of alcohols. It can be said that, at least partially, the convergence of Hungarian diet with the European one is bringing new issues with respect to the quality of the population's diet and its possible impacts on health.

Results are equally relevant for healthcare professionals, farmers, agro-food enterprises and different public bodies that need to know how much and what the population of a region or a country eats. Nutrition, or rather poor nutrition, is the main cause of morbidity and mortality in Europe and, consequently, successful nutritional policies might prove to be a fundamental step for the improvement of health in Europe. The success of these policies depends on a clear understanding of the dietary patterns of the population and how different socio-economic factors influence these patterns. This study hopefully adds to that understanding in the context of a European transition economy.

There is, of course, room for further research on this topic such as the comparison of the dietary changes in Hungary with the changes experienced by neighbouring and other European countries. It would also be interesting to analyse the Hungarian diet according to the WHO recommendations and to cross the data of the household panel with health data, most importantly with foodconsumption-related diseases such as obesity or coronary diseases. 


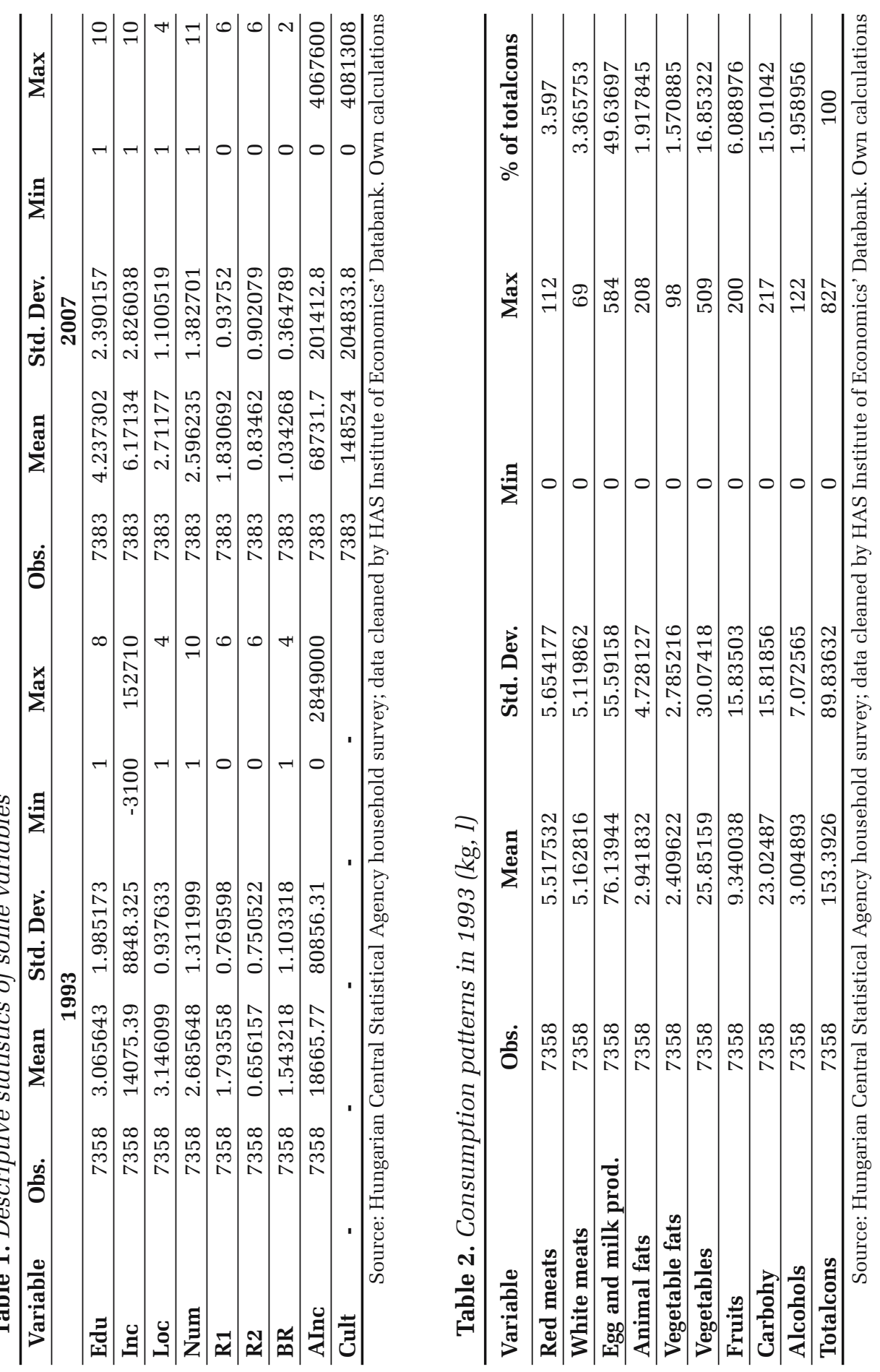



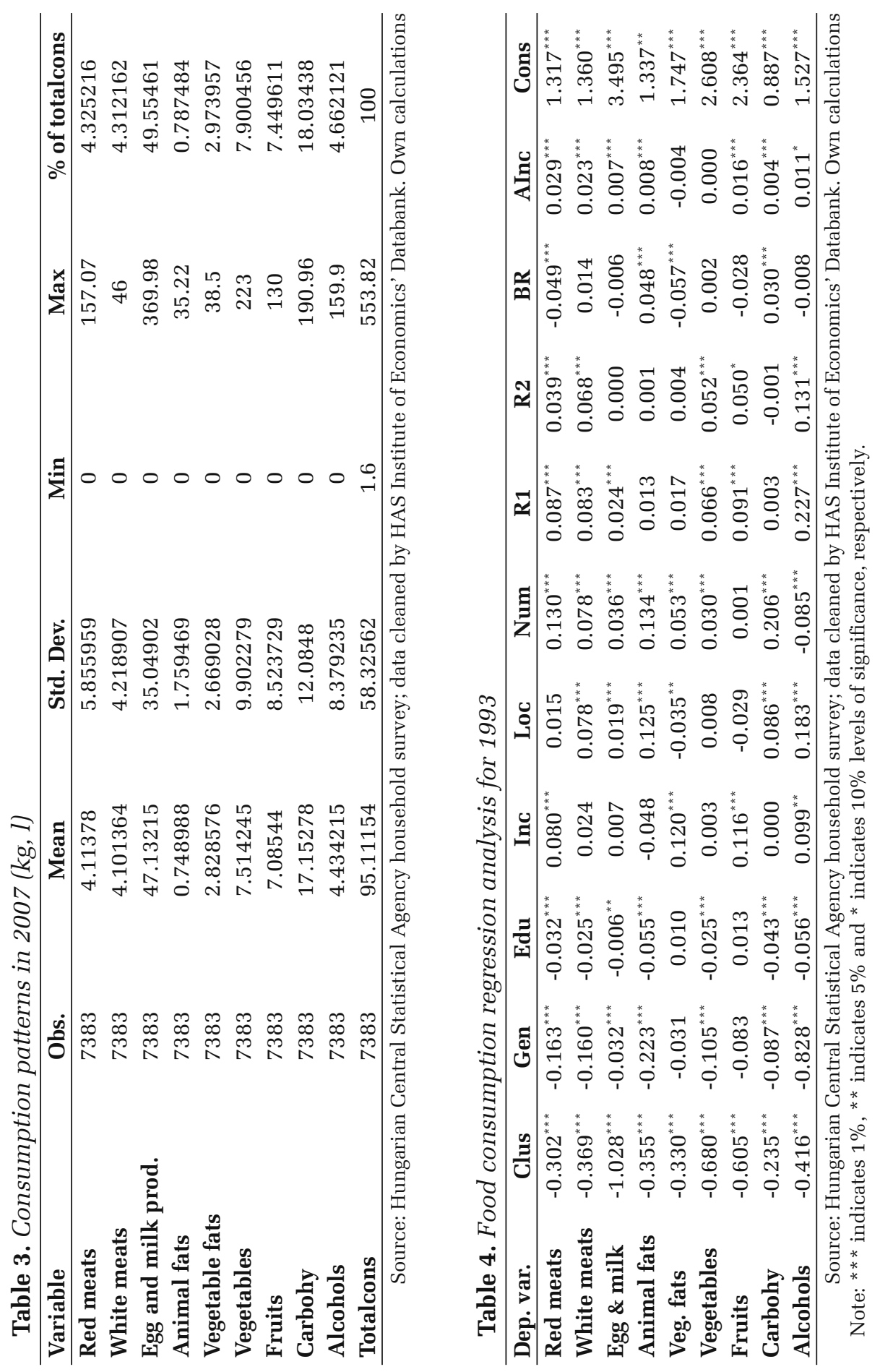


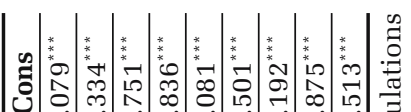

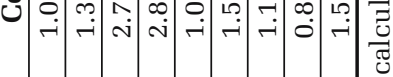

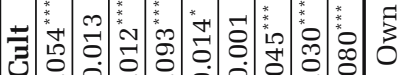

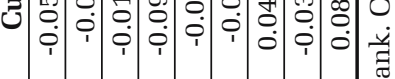

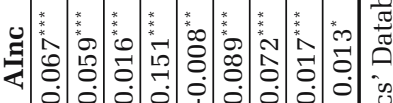

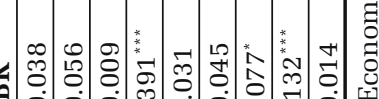

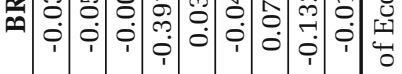

20

$\therefore=0: 0: 0: 0=0$

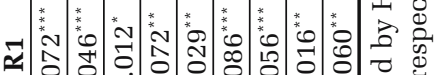

$=0: 0$

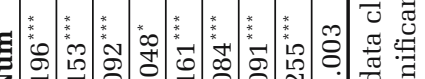

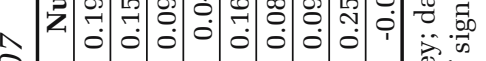

กิ

至

.

(3)

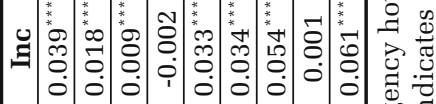

ฮี

की

Z

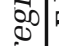

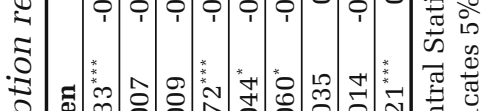

ㄴ.

పิ

ช

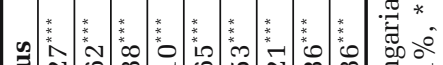

(-)

这

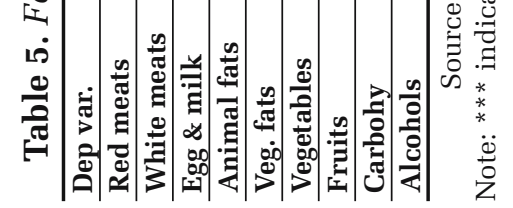

$\mid$

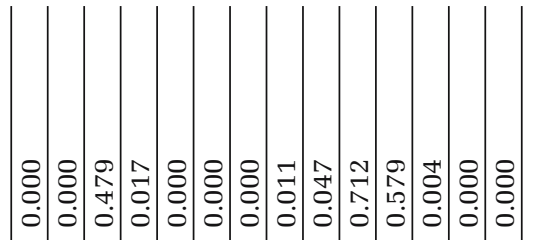

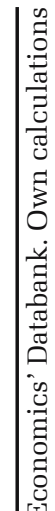

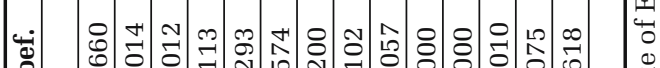

ن

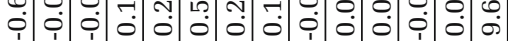

泀

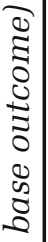

배욤

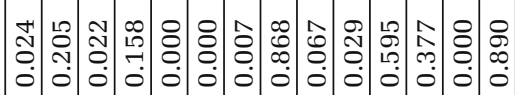

$\frac{\sqrt{5}}{\sqrt[5]{3}}$

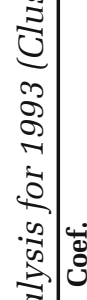

竎

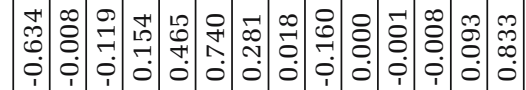

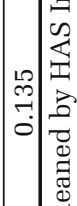

\%

营

:

离

כo

$\frac{4}{3}$

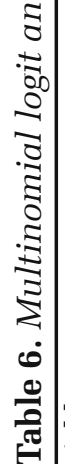


Table 7. Logit analysis for 2007 (Cluster 2 base outcome)

\begin{tabular}{|c|c|c|}
\hline Variables & Coef. & Signif. \\
\hline & \multicolumn{2}{|c|}{ Cluster 1} \\
\hline Gen & -0.326 & 0.000 \\
\hline Age & 0.013 & 0.000 \\
\hline Edu & -0.061 & 0.000 \\
\hline Inc & 0.031 & 0.030 \\
\hline Loc & 0.124 & 0.000 \\
\hline Num & 0.583 & 0.000 \\
\hline R1 & 0.172 & 0.000 \\
\hline R2 & 0.130 & 0.001 \\
\hline BR & -0.009 & 0.919 \\
\hline Cult & 0.009 & 0.719 \\
\hline Mealsg & -0.006 & 0.233 \\
\hline AInc & 0.161 & 0.000 \\
\hline Health & 0.062 & 0.000 \\
\hline cons & -4.192 & 0.000 \\
\hline Pseudo $^{2}$ & & \\
\hline
\end{tabular}

Source: Hungarian Central Statistical Agency household survey; data cleaned by HAS Institute of Economics' Databank. Own calculations

\section{References}

Adler, N.; Boyce, T.; Chesney, M.; Cohen, S.; Folkman, S.; Kahn, R.; Syme, S. (1994). Socio-economic status and health. Am. Psychology 49: 15-24.

Arija, V.; Salas-Salvado, J.; Fernandes-Ballart, J.; Cuco, G.; Marti-Henneberg, C. (1996). Consumo , hábitos alimentarios y estado nutricional de la poblacion de Reus. (IX). Med. Clin. (Barc) 106: 174-179.

Bourdieu, P. (1989). Distinction: a social critique of the judgment of taste. London, Routledge \& Kegan Paul.

Carels, R. A.; Konrad, K.; Harper, J. (2007). Individual differences in food perceptions and calorie estimation: An examination of dieting status, weight, and gender. Appetite 49: 450-458.

Cavelaars, A.; Kunst, A.; Mackenbach, J. (1997). Socio-economic differences in risk factors for morbidity and mortality in the European community: an international comparison. Journal of Health Psychology 2: 353-372.

Dowler, E.; Barlosius, E.; Feichtinger, E.; Kohler, B. (1997). Poverty, food and nutrition. In: B. M. Kohler, E. Feichtinger, E. Barlosius, E. Dowler (eds.), Poverty and Food in Welfare Societies. Berlin, Ramer Bohn, 17-32.

Grignon, C. (1999). Long-Term Trends in food consumption: a French portrait. Food Foodways 8 (3): 151-74. 
Hair, J. F. Jr.; Anderson, R.; Tatham, R.; Black, W. C. (1998). Multivariate Data Analysis. Upper Saddle River, NJ: Prentice Hall.

Hjartaker, A.; Lund, E. (1998). Relationship between dietary habits, age, lifestyle, and socio-economic status among adult Norwegian women. The Norwegian Women and Cancer Study. European Journal of Clinical. Nutrition 52: 565-572.

Hoeymans, N.; Smit, H.; Verkley, H.; Kromhout, D. (1996). Cardiovascular risk factors in relation to educational level in 36,000 men and women in the Netherlands. European Heart Journal 17: 518-525.

Huffman, S. K. (2005). Evaluation of consumption and welfare changes under economic transition in rural Poland. Eastern European Economics 43(3): 5-20.

Hulshof, K.; Brussaard, J.; Kruizinga, A.; Telman, J.; Lowik, M. (2003). Socioeconomic status, dietary intake and $10 \mathrm{y}$ trends: the Dutch National Food Consumption Survey. European Journal of Clinical Nutrition 57: 128-137.

Irala-Estevez, J.; Groth, M.; Johansson, L.; Prattala R.; Martınez-Gonzalez, M. (2000). A systematic review of socio-economic differences in food habits in Europe: consumption of fruit and vegetables. European Journal of Clinical Nutrition 54: 706-714.

Ivanova, L.; Dimitrov, P.; Ovcharova, D.; Dellava, J.; Hoffman, D. J. (2006). Economic transition and household food consumption: A study of Bulgaria from 1985 to 2002. Economics and Human Biology 4: 383-397.

James, W.; Nelson, M.; Ralph, A.; Leather, S. (1997). Socio-economic determinants of health. The contribution of nutrition to inequalities in health. British Medical Journal 314: 1545-1549.

Margetts, M.; Martinez, J.; Saba, A.; Holm, L.; Kearney, M. (1997). Definitions of 'healthy eating': a pan EU survey of consumer attitudes to food, nutrition and health. European Journal of Clinical. Nutrition 51: S23-S29.

Martınez-Gonzalez, M.; Lopez-Azpiazu, I.; Kearney, M.; Gibney, M.; Martınez, J. (1998). Definition of healthy eating in the Spanish adult population: a national sample in a pan-European survey. Public Health 112: 95-101.

Prattala, R.; Groth, M.; Oltersdorf, U.; Roos, G.; Sekula,W.; Tuamainen, H. (2003). Use of butter and cheese in 10 European countries. A case of contrasting educational differences. European Journal of Public Health 13 (2): 124-32.

Provencher, V.; Polivy, J.; C. Peter Herman, C. P. (2008). Perceived healthiness of food. If it's healthy, you can eat more! Appetite 52: 340-344.

Regmi, A.; Takeshima, H.; Unnevehr, L. (2008). Convergence in global food demand and delivery. Economic Research Report No. 56, Economic Research Department, USDA.

Regmi, A.; Gehlhar, M. (2005). New directions in global food markets. AIB - 794, Economic Research Service, U.S.D.A. 
Roos, G.; Prattala, R. and the FAIR-97-3096 Disparities Group (1999). Disparities in food habits. Review of Research in 15 European Countries. Helsinki, National Public Health Institute.

Roos, G.; Prattala, R.; Lahelma, E.; Kleemola, P.; Pietinen, P. (1996). Modern and healthy? Socioeconomic differences in the quality of diet. European Journal of Clinical Nutrition 50: 753-60.

Seale, J.; Regmi, A.; Bernstein, J. (2003). International evidence on food consumption patterns. Technical Bulletin No. 1904, Economic Research Service, U.S.D.A. 